\title{
Tratamiento de schwanomas vestibulares esporádicos mediante radiocirugía: Estudio prospectivo de cohorte
}

\section{Radiosurgical treatment of sporadic vestibular schwannomas : A prospective cohort study}

Freddy Martel V1', Rodrigo Iñiguez S1', Daniel Venencia M², Patricio Tagle M², Pelayo Besa D³, José Lorenzoni S².

\section{RESUMEN}

Objetivo: Analizar los resultados iniciales de un estudio prospectivo en pacientes con schwanomas vestibulares tratados con radiocirugía en la Pontificia Universidad Católica de Chile.

Material y método: Se presentan 17 pacientes portadores de schwanomas vestibulares esporádicos tratados con radiocirugía y seguidos entre 6 y 26 meses. $\theta$ tratamiento fue realizado con acelerador lineal Varian clinac $21 \mathrm{EX}$, con equipamiento Varian-Zmed. La dosis marginal administrada al tumor fue de 12 a 12,5 Gray. I seguimiento a 6, 12 y 24 meses consta de resonancia magnética, audiometría y evaluación clínica

Resultados: Ladosis marginal de irradiación usada fue entre 12 y 12,5 Gay normalizadaa la isodosis $70 \%$ u $80 \%$. En todos los pacientes se documentó disminución de la captación de contraste del tumor y en 16 (94\%) se observaron áreas de necrosis centro tumoral. No hubo mortalidad, la preservación de audición útil fue 62,5\% actuarial a 2 años. No ha existido deterioro de la función de los nervios facial ni trigémino. Todos los pacientes que previamente estaban trabajando retornaron a sus labores en promedio 11,5 días luego del tratamiento.

Conclusiones: Los resultados iniciales de esta serie son comparables a los resultados publicados en la literatura y refuerzan el demostrado rol de la radiocirugía en el tratamiento de los schwanomas vestibulares.

Palabras clave: Schwanomas, radiocirugía, acelerador lineal.

\section{SUMMARY}

Objective: To analyze the preliminary experience of radiosurgery for Vestibular Schwannomas at the Pontificia Universidad Católica de Chile.

Material and methods: The first 17 patients with sporadic Vestibular Schwannomas treated by radiosurgery at our institution are reported. The marginal dose used was 12 to 12.5 Gy. prescribed at the 70 or 80 isodose line. Patients were controlled at 6, 12 and 24 months with magnetic resonance, audiometric study and clinical examination.

Results: In all of the 17 patients treated a decrease tumor enhancement on MR was demonstrated. In 16 patients (94\%) a pattern of central tumor necrosis was observed during the firs year. Actuarial useful hearing was maintained in $62.5 \%$ at 2 year after treatment. Facial nerve function was maintained in all of the 15 patients with normal function at treatment (100\%). Trigeminal function was maintained in all of the 14

1Departamento de Otorrinolaringología, '2Departamento de Neurocirugía y Departamento de Radiología (Servicio de Radioterapia $)^{3}$, Escuela de Medicina, Pontificia Universidad Católica de Chile. 
patients (100\%) with previous normal trigeminal function. The mean time to return to work or normal activities was 11.5 days after treatment

Conclusions: These preliminary results are comparable with results published in the literature and reinforce the demonstrate role of radiosurgery in the management of vestibular schwannomas.

Key words: Schwannomas, radiosurgery, linear accelerator.

\section{INTRODUCCIÓN}

El schwanoma vestibular (SV) es un tumor benigno, originado en las células de Schwann, que forman la vaina de mielina que rodea las ramas vestibulares del octavo nervio craneano.

Tiene una incidencia estimada en 0,7 casos por cada 100.000 habitantes por año $0^{1,2}$, representando entre $6 \%$ y $10 \%$ de los tumores primarios intracraneanos. Es el tumor más común del ángulo pontocerebeloso ${ }^{3}$ y se presenta con mayor frecuencia entre la cuarta y sexta década de la vida.

La sintomatología habitual es hipoacusia unilateral progresiva $(80 \%)$, tinnitus $(50 \%)$ y vértigo $(30 \%)$. En caso de tumores de mayor tamaño se agrega compromiso del nervio facial, trigémino y del tronco cerebral ${ }^{4}$.

El diagnóstico se realiza principalmente con resonancia magnética de cerebro, la cual muestra en la secuencia $\mathrm{T} 1$ una imagen isointensa que se hace fuertemente hiperintensa con el uso de medio de contraste ${ }^{5,6}$. Actualmente por la masificación y el mayor acceso de los pacientes a estudios neurorradiológicos se diagnostican tumores cada vez más pequeños, oligosintomáticos y en algunos casos asintomáticos.

Los objetivos del tratamiento del schwanoma vestibular son: preservar la vida del paciente, controlar el crecimiento tumoral, mantener la función de los nervios craneanos (facial, trigémino, glosofaríngeo) e idealmente preservar la función auditiva.

La conducta frente a un schwanoma vestibular puede ser: observación $n^{7-9}$, microcirugía ${ }^{4,10-15}$, y radiocirugía ${ }^{16-43}$.

La radiocirugía en SV data del año 1969 gracias a la genialidad del neurocirujano sueco Lars Leksel ${ }^{44}$. Consiste en administrar en una sesión múltiples haces de radiación provenientes desde distintos puntos dirigidos en forma convergente al tumor, usando técnica estereotáxica para su localización. De esta forma, la dosis de radiación recibi- da en el tumor es alta siendo baja en los tejidos sanos circundantes.

Existe hoy en día suficiente respaldo en la literatura para el tratamiento de schwanomas vestibulares con radiocirugía ya sea realizada con Gamma Knife $16,17,28,29,31-34,38-41$, así como también radiocirugía realizada con acelerador lineal (Linac) $)^{20-26,42}$. En ambas modalidades de tratamiento se ha logrado alto control tumoral en el largo plazo, del orden de $90 \%$ o más en seguimientos de hasta 15 años ${ }^{20,27}$.

En un comienzo se utilizó dosis altas de irradiación (20-30 Gy), con lo cual se obtenía buen control tumoral pero alto porcentaje de neuropatía auditiva, facial y trigeminal ${ }^{21,29,42}$. Actualmente, la dosis marginal de tratamiento recomendada es menor, en el rango de 12-13 Gray ${ }^{20-22,34,36}$, con lo que se mantiene el efecto antitumoral, pero disminuyen considerablemente los riesgos de neuropatías, lográndose mantener audición útil en el largo plazo en rangos de $42 \%$ a $88 \%{ }^{17,21-23,25,26,29,31-34,36-39,43}$ y preservando al máximo la función de los nervios facial y trigémino ${ }^{17,20,22,23,25,36,32,34,38,30}$.

El objetivo de este estudio es evaluar en forma preliminar los resultados de la radiocirugía en pacientes portadores de schwanomas vestibulares esporádicos tratados en la Pontificia Universidad Católica de Chile con énfasis en: control tumoral, respuesta radiológica, preservación de la audición, preservación de la función de los nervios facial, y trigémino y el tiempo de retorno al trabajo de los pacientes tratados. También se analizan las eventuales complicaciones del tratamiento.

\section{MATERIAL Y MÉTODO}

Se realizó un estudio prospectivo que incluyó 17 pacientes portadores de schwanoma vestibular esporádico, tratados mediante radiocirugía estereotáxica en el Centro de Cáncer de la Pontificia Universidad 
Católica de Chile durante el período comprendido entre marzo de 2006 y enero de 2008.

Los pacientes presentaban tumores inferiores a 3 centímetros de diámetro y sin efecto de masa importante sobre el tronco cerebral (grados $1 \mathrm{a} 4 \mathrm{a}$ de Koos $)^{45}$. Fueron excluidos del estudio aquellos pacientes portadores de neurofibromatosis tipo $2 \mathrm{y}$ pacientes con seguimiento inferior a 6 meses.

Previo al tratamiento todos los pacientes fueron estudiados con examen clínico, resonancia magnética cerebral y prueba de octavo par.

El tratamiento se realizó con acelerador lineal VARIAN ${ }^{\circledR} 21$ EX, usando fotones de 6 megaelectronvolts. Se usó el sistema de radiocirugía VARIAN-Zmed ${ }^{\circledR}$. La planificación del tratamiento se hizo en base a resonancia magnética de 1,5 TESLA empleando cortes de $1 \mathrm{~mm}$ de espesor y tomografía computarizada de cerebro de alta resolución obtenida en tomógrafo helicoidal de 64 canales con cortes de 1 $\mathrm{mm}$ de espesor.

La radiocirugía fue realizada por un equipo multidisciplinario constituido por un neurocirujano, un radioterapeuta y un físico médico.

Se usó dosis marginal (envolvente) entre $12 \mathrm{y}$ 12,5 Gray, normalizada a la curva de isodosis $70 \%$ u $80 \%$.

El protocolo de tratamiento se ciñó a las recomendaciones de la RTOG (Radiotheraphy Oncology Group) en lo que se refiere a calidad del equipamiento empleado, controles de calidad y estrategias de planificación ${ }^{46}$.

Al inicio de la experiencia clínica el equipamiento de radiocirugía fue sometido a una auditoría internacional voluntaria realizada por un organismo oficial de la RTOG (MD Anderson Cancer Center, University of Texas), en el cual se constató que todos los parámetros de precisión mecánica y dosimétrica de las instalaciones estaban acordes con las recomendaciones de la RTOG para realizar radiocirugía ${ }^{47}$.

Previo a cada tratamiento se chequeó la calibración del equipo según protocolos estándares establecidos. En lo que se refiere a conformación, la planificación del tratamiento se realizó acorde a las recomendaciones de la $\mathrm{RTOG}^{46}$, de manera tal de administrar la radiación ceñida a la forma tridimensional del tumor. Para este efecto, se usó el índice de conformación ${ }^{46}$ definido como el cuociente entre el volumen de tejido irradiado a la dosis de prescripción y el volumen del tumor. Se consideró como valor deseable un índice inferior a 2.

El tratamiento fue ambulatorio en la mayoría de los casos. En 4 pacientes se realizó hospitalización de una noche a solicitud de los propios pacientes por razones de mejor cobertura económica por parte de sus sistemas previsionales de salud.

Los controles posteriores al tratamiento fueron programados a los 6 y 12 meses y luego anualmente, e incluyeron examen clínico, resonancia magnética de encéfalo y audiometría.

La función auditiva fue evaluada en aquellos pacientes que previo al tratamiento presentaran audición útil según la escala de Gardner-Robertson ${ }^{48}$, que considera como audición útil los grados I y II (audición tonal promedio de 50 decibeles o menos en frecuencias de 500 a 2.000 Hertz y discriminación de $50 \%$ o más). Para representar la preservación de la audición útil en el tiempo, se usó el método de curva actuarial de Kaplan-Meier.

La función del nervio facial se evaluó en los pacientes que al momento del tratamiento tuvieran función indemne, de acuerdo a la escala de HouseBrackman ${ }^{49}$, se consideró como función normal el grado 1.

La función trigeminal se evaluó en pacientes con indemnidad trigeminal previa al tratamiento y fue valorada en forma clínica.

Se consideró como respuesta tumoral radiológica inicial (durante el primer año) la disminución en la captación de medio de contraste por el tumor y/o la consolidación de áreas de necrosis intratumoral en la resonancia magnética. Fue definido como control tumoral la ausencia de crecimiento ulterior del tumor y sin necesidad de cirugía.

\section{RESULTADOS}

En el período comprendido entre marzo de 2006 y enero de 2008, 17 pacientes portadores de schwanomas vestibulares esporádicos fueron tratados mediante radiocirugía estereotáxica.

Las Tablas 1 y 2 resumen las características generales de los pacientes, del tratamiento y los resultados.

La serie comprende 13 hombres y 4 mujeres con una edad promedio de 51 años (40-71), portadores 
Tabla 1. Características generales de los pacientes de la serie

\begin{tabular}{|ccccccccc|}
\hline Paciente & Sexo & $\begin{array}{c}\text { Edad } \\
\text { (años) }\end{array}$ & Lado & $\begin{array}{c}\text { Volumen } \\
\text { (cc) }\end{array}$ & $\begin{array}{c}\text { Grado } \\
\text { (Koos) }\end{array}$ & $\begin{array}{c}\text { Cx. } \\
\text { previa }\end{array}$ & $\begin{array}{c}\text { Dosis } \\
\text { (Gray) }\end{array}$ & $\begin{array}{c}\text { Seguimiento } \\
\text { (meses) }\end{array}$ \\
\hline 1 & $\mathrm{~F}$ & 44 & Izq & 0,8 & 3 & No & 12 & 26 \\
2 & $\mathrm{~F}$ & 57 & Der & 3,4 & 3 & No & 12,5 & 24 \\
3 & $\mathrm{M}$ & 44 & Izq & 0,14 & 1 & No & 12,5 & 26 \\
4 & $\mathrm{M}$ & 60 & Der & 4 & $4 \mathrm{a}$ & No & 12 & 24 \\
5 & $\mathrm{M}$ & 54 & Izq & 1,05 & 2 & No & 12,5 & 25 \\
6 & $\mathrm{M}$ & 42 & Izq & 0,35 & 2 & No & 12,5 & 26 \\
7 & $\mathrm{M}$ & 49 & Der & 3,1 & 3 & Si & 12,5 & 25 \\
8 & $\mathrm{M}$ & 49 & Izq & 1,9 & 3 & No & 12,5 & 24 \\
9 & $\mathrm{M}$ & 43 & Der & 5,4 & 3 & No & 12,5 & 15 \\
10 & $\mathrm{~F}$ & 66 & Der & 2,5 & 3 & Si & 12,5 & 12 \\
11 & $\mathrm{M}$ & 50 & Izq & 0,4 & 2 & No & 12,5 & 12 \\
12 & $\mathrm{~F}$ & 71 & Izq & 0,6 & 3 & No & 12,5 & 12 \\
13 & $\mathrm{M}$ & 64 & Der & 1,8 & 3 & No & 12,5 & 12 \\
14 & $\mathrm{M}$ & 45 & Der & 5,3 & 4 a & No & 12,5 & 6 \\
15 & $\mathrm{M}$ & 48 & Der & 7 & $4 \underline{\underline{a}}$ & No & 12 & 6 \\
16 & $\mathrm{M}$ & 41 & Izq & 0,2 & 1 & No & 12,5 & 6 \\
17 & $\mathrm{M}$ & 40 & Der & 1,2 & 2 & No & 12,5 & 6 \\
\hline
\end{tabular}

Tabla 2. Resumen de los resultados obtenidos en los pacientes tratados

$\begin{array}{ll}\text { Edad ( años) } & \text { Promedio: 51/rango: } 40 \text { a } 71 \\ \text { Volumen tumoral (cc) } & \text { Promedio: 2,3/rango: } 0,14-7 \\ \text { Dosis marginal (Gray) } & \text { Promedio: } 12,4 / \text { rango: } 12-12,5 \\ \text { Isodosis de prescripción (\%) } & \text { Promedio: } 73 / \text { rango: } 70-80 \\ \text { Número de isocentros } & \text { Promedio: } 2,4 / \text { rango: } 1-4 \\ \text { Índice de conformación } & \text { Promedio: } 1,7 / \text { rango: } 1,2-2,8 \\ \text { Seguimiento (meses) } & \text { Promedio: } 16,9 / \text { rango: } 6-26 \\ \text { Mortalidad } & 0 / 17(0 \%) \\ \text { Control tumoral } & 17 / 17(100 \%) \\ \text { Menor captación de contraste } & 17 / 17(100 \%) \\ \text { Necrosis centro tumoral } & 16 / 17(94 \%) \\ \text { Preservación de audición útil } & 62,5 \% \text { actuarial a } 26 \text { meses } \\ \text { Preservación de nervio facial } & 15 / 15(100 \%) \\ \text { Preservación de nervio trigémino } & 14 / 14(100 \%) \\ \text { Reintegro laboral (días) } & \text { Promedio: } 11,5 / \text { rango: } 2-30\end{array}$

de tumores de 2,3 cc de promedio (0,14-7 cc). Dos pacientes presentaban recurrencia posterior a cirugía y en 15 pacientes la radiocirugía fue el primer tratamiento. Todos los pacientes tuvieron seguimiento prospectivo y control actualizado al momento del cierre del estudio. El seguimiento promedio fue de 16,9 meses con un rango de 6 a 26 meses.

En todos los pacientes hubo control tumoral y en todos se documentó disminución en la captación de contraste en el tumor durante los primeros meses
(Figuras 2 y 3). En 16 pacientes (94\%) se observó consolidación de áreas de necrosis intratumoral. En un caso no se observó necrosis, se trata de un paciente de 44 años portador de un pequeño tumor intracanalicular de 0,14 cc en el que sí se demostró disminución en la captación de medio de contraste.

No hubo mortalidad. Entre los 15 pacientes con función normal del nervio facial al momento del tratamiento, ninguno presentó deterioro postratamiento. Tampoco hubo deterioro de la función del 


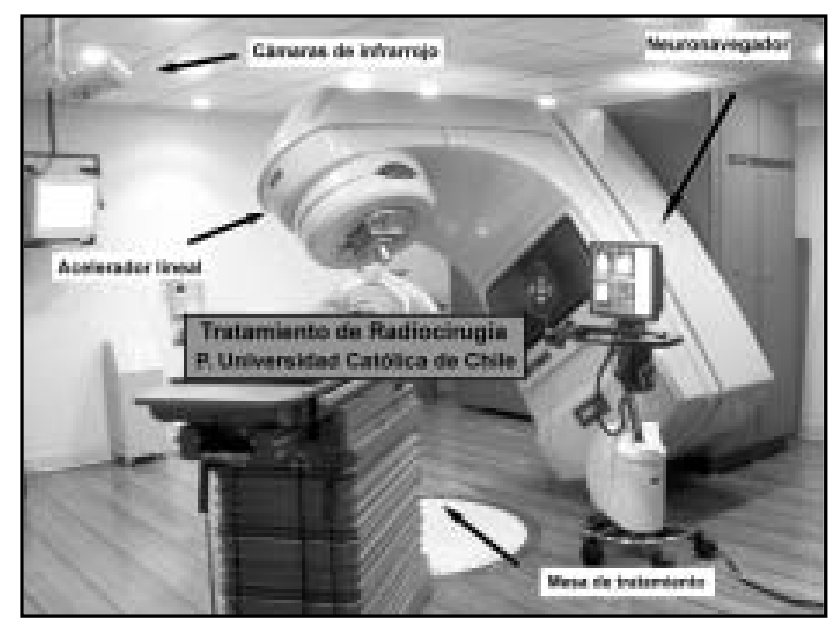

Figura 1. Equipamiento para radiocirugía usado en el Centro de Cáncer de la Pontificia Universidad Católica de Chile. Se compone de un acelerador lineal (Linac) Varian modelo 21 EX, equipado con sistema de radiocirugía Varian-Zmed. Durante el tratamiento el acelerador lineal y la mesa de tratamiento rotan en forma isocéntrica de manera tal de generar arcos de irradiación concéntricos no coplanares. El posicionamiento del paciente es monitorizado en tiempo real mediante neuronavegación durante todo el tratamiento

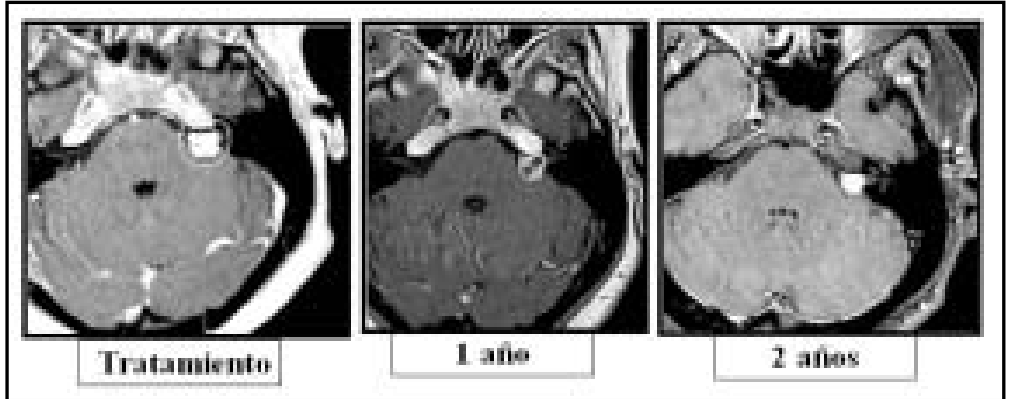

Figura 2. Ejemplo de paciente de sexo femenino de 44 años de edad con hipoacusia izquierda marcada (Gardner-Robertson III), tinnitus y síndrome vertiginoso. Se puede observar disminución en la captación de medio de contraste en el centro del tumor al año del tratamiento e involución tumoral a los 2 años.

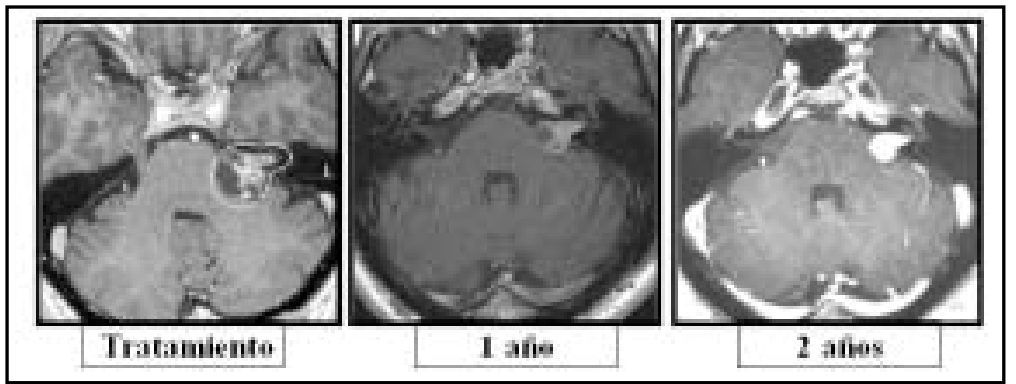

Figura 3. Ejemplo de paciente de sexo masculino de 60 años de edad con hipoacusia izquierda leve (Gardner-Robertson I) y tinitus. Se puede observar disminución en la captación de medio de contraste en el centro del tumor y disminución del tamaño de los quistes intratumorales al año del tratamiento e involución tumoral a los 2 años con desaparición del componente quístico. A los 2 años de tratamiento el paciente presenta audición útil (Gardner-Robertson II).

nervio trigémino en los 14 pacientes que presentaban función trigeminal normal al ser tratados. En cuanto a la función auditiva, 10 pacientes presentaban audición útil al momento del tratamiento (grados I ó II de Gardner-Robertson), durante el seguimiento, 3 pacientes $(30 \%)$ perdieron la audición útil pasando a grado III de la escala. La preservación actuarial de audición útil fue de 62,5\% a dos años (Figura 4).
Cuatro pacientes presentaron sensación de mareos $y / 0$ vértigos de magnitud leve a moderada y en forma transitoria después de la radiocirugía. En todos ellos el tratamiento consistió en reposo y en 2 pacientes se indicó vasodilatadores. En aquellos pacientes que previamente estaban trabajando el reintegro a su trabajo fue en promedio a los 11,5 días (2-30), (en 2 pacientes la sensación vertiginosa retrasó el reintegro laboral a 30 días postratamiento). 
Figura 4. Gráfico que muestra en forma actuarial la preservación de audición útil (Gardner-Robertson I ó II) en 10 de los 17 pacientes de la serie que al momento de la radiocirugía presentaban audición útil.

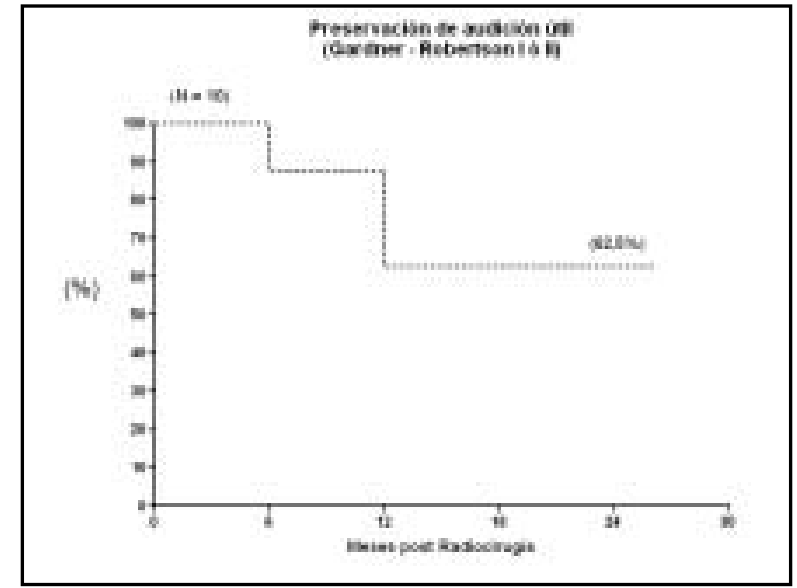

microangiopatía tumoral radioinducidas, se produce en el interior del estroma tumoral proliferación de fibroblastos reparativos que llevan a una esclerosis tumoral ${ }^{50,51}$.

Los seguimientos más largos de pacientes portadores de schwanomas vestibulares tratados con radiocirugía reportan control tumoral actuarial entre $87 \%$ y $97 \%$ a 10 y 15 años $20,27,29,32,30$, definiéndose como control tumoral la ausencia de crecimiento ulterior del tumor y el no ser necesaria la cirugía.

Estudios más recientes, usando las dosis marginales recomendadas hoy en día (12 a 13 Gy), comunican tasas de control tumoral de $92 \%$ a 5 años $^{34}$ y $98,6 \%$ a 6 años ${ }^{36}$.

En nuestra serie, todos los pacientes fueron tratados con dosis según lo recomendado hoy en día (12 a 13 Gy.) y, si bien, el seguimiento es corto (entre 6 y 26 meses), no se ha documentado falla de tratamiento y ningún paciente ha requerido cirugía.

Las principales complicaciones que se intenta evitar o reducir con la radiocirugía son la neuropatía facial, la neuropatía trigeminal y el deterioro de la audición. Tales riesgos son dependientes de la dosis de radiación usada y del tamaño del tumor.

Ninguno de los pacientes tratados en este estudio ha presentado deterioro de la función de los nervios facial ni trigémino. Empleando dosis marginal en el rango recomendado hoy en día, (12 a 13 Gray) el riesgo de dañar irreversiblemente el nervio facial es menor a $1 \%{ }^{20,23,34,36}$. Spigelman ${ }^{21}$ comunica que la neuropatía facial se manifiesta dentro del primer año luego del tratamiento. 
El riesgo de neuropatía trigeminal permanente reportado con las dosis actualmente recomendadas es entre $0 \%$ y $4 \%{ }^{20,23,34,36}$.

La preservación de la audición útil definida como categorías I ó II en la escala de GardnerRobertson es otro punto importante que se persigue con la radiocirugía. En el presente estudio, de los 10 pacientes con audición útil al momento del tratamiento, $3(30 \%)$ deterioran su audición a un nivel considerado no útil (categoría 3 en la escala de Gardner y Robertson). Ningún paciente presentó pérdida absoluta de su audición. Al usar curvas de Kaplan-Meier, la mantención de audición útil a 2 años (curva actuarial) fue de $62,5 \%$.

La probabilidad reportada en la literatura de mantener audición útil en el largo plazo varía entre $42 \%$ y $88 \% 17$ 17,21-23,25,26,29,31-34,36-39,43, dependiendo fundamentalmente del tamaño del tumor, la dosis utilizada y tiempo de seguimiento.

Bush $^{43}$ en una serie de 17 pacientes tratados con Gamma knife usando dosis marginal promedio de 13,8 Gy observa $42 \%$ de preservación de audición útil, Paek ${ }^{38}$, reporta $52 \%$ de conservación de audición útil en un estudio de 25 pacientes tratados con Gamma Knife con una dosis promedio de 12 Gy.

Massager ${ }^{39}$ comunica $56 \%$ de preservación de audición útil actuarial a 4 años en un estudio en 82 pacientes tratados con Gamma Knife con una dosis de 12 Gy. En tal estudio se observa deterioro gradual de audición luego del tratamiento, más marcado en los primeros 2 años.

Flickinger ${ }^{36}$ obtiene $78 \%$ de preservación de audición útil actuarial a 5 años en un estudio que incluye 313 pacientes tratados con Gamma Knife con dosis de 12 a 13 Gy.

El estudio más numeroso publicado sobre schwanomas vestibulares tratados con radiocirugía corresponde a la serie de Régis et al ${ }^{17}$. Tal estudio comprende 1.500 pacientes tratados con Gamma Knife con dosis marginal de 12 Gy. La preservación de audición útil fue de $60 \%$.

En lo que se refiere a tratamientos realizados con acelerador lineal (Linac), Spiegelmann ${ }^{22}$ en un estudio que incluye 318 pacientes tratados con una dosis promedio de 12,5 Gy y seguimiento promedio de 44 meses, comunica preservación de audición útil en el $62 \%$ de los pacientes. Wurm ${ }^{23}$, reporta preservación de audición útil actuarial a 5 años de $87 \%$ en una serie de 64 pacientes tratados con una dosis de 12 Gy.

El riesgo de deterioro de la audición posterior a la radiocirugía ha sido relacionado con el volumen del componente intracanalicular del tumor ${ }^{39}$ y con la dosis de radiación recibida por la cóclea ${ }^{38,40,41}$.

La morbimortalidad, calidad de vida y reintegro laboral de los pacientes tratados con radiocirugía al igual que los costos globales del tratamiento han sido estudiados. Se ha comunicado ventajas significativas de la radiocirugía respecto a la cirugía convencional ${ }^{16,18,19}$. Regis ${ }^{16}$ reporta un reintegro laboral en el $99 \%$ de los pacientes tratados con radiocirugía en un plazo de 7 días.

No hay publicaciones de mortalidad asociada al procedimiento de radiocirugía en schwanomas vestibulares, sin embargo se ha comunicado 2 casos de transformación maligna de schwanoma vestibular tratados con radiocirugía ${ }^{53,54}$.

En schwanomas vestibulares quísticos se han comunicado casos de fracaso del tratamiento con radiocirugía ${ }^{55,56}$, con crecimiento ulterior del quiste, de modo que, en tumores quísticos, la cirugía convencional podría ser una mejor opción terapéutica.

Si bien es cierto, la radiocirugía permite una alta probabilidad de control tumoral ( $90 \%$ a $98 \%)$, en aquellos casos en que la radiocirugía no logra controlar el tumor la cirugía convencional subsecuente podría ser más dificultosa. Shuto ${ }^{56}$ reporta una serie de 12 pacientes portadores de schwanoma vestibular operados luego de un tratamiento fallido de radiocirugía con Gamma Knife. En 3 casos el aumento del tumor se debió a aumento de componente quístico, en 2 casos a necrosis y en 7 casos a aumento de componente sólido. Se encontró escaso sangramiento quirúrgico en casi todos los pacientes, sin embargo se comunica dificultad de la cirugía debido a adherencias del tumor al tronco cerebral y a nervios craneanos. El autor recomienda realizar resección subtotal sin disecar el nervio facial ya que el crecimiento ulterior del residuo tumoral fue raro.

La radiocirugía ha sido utilizada en pacientes portadores de neurofibromatosis tipo 2, con resultados algo inferiores en términos de control tumoral, neuropatía facial, trigeminal y preservación de audición respecto a aquellos pacientes con tumores esporádicos ${ }^{30,57}$. Los pacientes portadores 
de esta enfermedad genética autonómica dominante presentan habitualmente múltiples tumores y sus expectativas de vida en el mediano plazo son limitadas.

En nuestra institución hemos tratado 2 pacientes con neurofibromatosis tipo 2, ambos pacientes con tumores bilaterales, portadores de otros tumores intracraneanos y espinales, múltiples cirugías previas $y$ en relativas pobres condiciones neurológicas. En ellos, el tratamiento de radiocirugía se realizó en un contexto paliativo. Ambos pacientes no fueron incluidos en el presente estudio, el cual se refiere a schwanomas vestibulares esporádicos tratados con intención curativa y en el contexto de un estudio de cohorte prospectivo planificado a largo plazo.

En conclusión, en la evaluación preliminar de esta pequeña serie de 17 pacientes tratados con radiocirugía, con un seguimiento relativamente corto (entre 6 a 26 meses), se han documentado los cambios tumorales radiológicos radioinducidos descritos en la literaratura y ha habido control tumoral en todos los pacientes. No ha habido morbilidad de nervios facial ni trigémino y la preservación actuarial de audición útil a 2 años ha sido $62,5 \%$. Estos resultados si bien preliminares, son acordes con lo publicado en la literatura.

\section{BIBLIOGRAFÍA}

1. Cohen R. Acoustic neuroma: summary of the NIH consensus. MD Med J 1992; 41: 1128-30.

2. Propp J, McCarthy B, Davis F et al. Descriptive epidemiology of vestibular schwannomas. Neuro-oncol 2006; 8: 1-11.

3. Lanser M, Sussman S, Frazer K. Epidemiology, pathogenesis and genetics of acoustic tumors. Otolaryngol Cin North Am 1992; 25: 499-520.

4. Samil M, Gerganov V and Samil A. Improved preservation of hearing anf facial nerve function in vestibular schwannoma surgery via the retrosigmoid approach in a series of 200 patients. J Neurosurg 2006; 105: 527-35.

5. SWARTZ J. Lesions of the cerebellopontine angle and internal auditory canal: diagnosis and differential diagnosis. Semin Ultrasound CT MR2004; 25: 332-52.
6. Walsh R, Bath A, Bance M et al. Comparison of two radiologic methods for measuring the size and growth rate of extracanalicular vestibular schwannomas. Am J Otol 2000; 21: 716-21.

7. Smouna E, Yoo M and Mohr K et al. conservative management of acoustic neuroma: a metaanalysis and proposed treatment algorithm. Laringoscope 2005; 115: 450-4.

8. Yоsнімото Y. Systematic review of the natural history of vestibular schwannoma. J Neurosurg 2005; 103: 59-63.

9. Yamakami I, Uchino $Y$, Kobayashi E and Yamamura $A$. Conservative management, Gamma Knife radiosurgery and microsurgery for acoustic neurinomas: A systematic review of outcome and risk of three therapeutic options. Neurological Research 2003; 25: 682-90.

10. Friedman R, Kesser B, Brackmann et al. Long-term hearing preservation after middle fossa removal of vestibular schwannoma. Aolaryngol Head Neck Surg 2003; 129: 660-5.

11. Colletti V, Fiorino F. Is the middle fossa approach the treatment of choise for intracanalicular vestibular schwannoma? Otolaryngol Head Neck Surg 2005; 132: 45966.

12. Miyakazi H, Deveze A, Magnan J. Neuro-otologic surgery through minimally-invasive retrosigmoid approach: endoscope-assisted microvascular decompression, vestibular neurotomy and tumor removal. Laringoscope 2005; 115: 1612-17.

13. Mohr G, Sade B, Dufour J et al. Preservation of hearing in patients undergoing microsurgery for vestibular schwannoma: degree of meatal filling. J Neurosurg 2005; 102: 1-5.

14. Lanman T, Brackmann D, Hitselberger W et al. Report of 190 consecutive cases of large acoustic tumors (vestibular schwannoma) removed via the translabyrinthine approach. $J$ Neurosurg 1999; 90:617-23.

15. Mamikoglu B, Wiet R, Esquivel C. Translabyrinthine approach for the management of large and giant vestibular schwannomas. Ool Neurotol 2002; 23: 224-27.

16. Régis J, Pellet W, Delsanti C et al. Functional outcome after Gamma Knife surgery or microsurgery for vestibular scwwannomas. $J$ Neurosurg 2002; 97: 1091-100. 
17. Régis J, Roche PH, Delsanti C et al. Modern management of vestibular schwannomas. Prog Neurol Surg 2007; 20: 129-41.

18. Karpinos M, The B, Zeck 0 et al. Treatment of acoustic neuroma: stereotactic radiosurgery vs. microsurgery. Int $J$ Radiation Oncology Biol Phys 2002; 54: 1410-21.

19. Myrseth E, Moller P, Pedersen P et al. Vestibular schwannomas: Clinical results and quality of life after microsurgery or Gamma Knife radiosurgery. Neurosurgery 2005; 56: 927-35.

20. Friedman W, Bradshaw P, Myers A et al. Linear accelerator radiosurgery for vestibular schwannomas. J Neurosurg 2006; 105: 657-61.

21. Spiegelman R, Lidar Z, Gofman J et al. Linear accelerator radiosurgery for vestibilar schwannoma. J Neurosurg 2001; 94: 7-13.

22. Spiegelman R, Menhel J, Alezra D et al. Linac radiosurgery for vestibular schwannomas. $8^{\text {th }}$ International Stereotactic Radiosurgery Society Congress, San Francisco, California, USA, June 2007: pag 156, (Abstract).

23. Wurm R, Scheffller D, Schlenger L et al. Conformal stereotactic radiosurgery for vestibular schwannoma. $8^{\text {th }}$ International Stereotactic Radiosurgery Society Congress, San Francisco, California, USA, June 2007: pag 204, (Abstract).

24. Okunaga T, Matsuo T, Hayashi N et al. Linear accelerator radiosurgery for vestibular schwannoma: measuring tumor volume changes on serial three-dimensional spoiled gradient-echo magnetic resonance images. $J$ Neurosurg 2005; 103: 53-8.

25. Combs S, Thilmann C, Debus J et al. Long-term outcome of stereotactic radiosurgery (SRS) in patients with acoustic neuromas. Int $J$ Radiation Oncology Biol Phys 2006; 64: 1341-7.

26. Meijer 0, Vandertop W, Baayen J et al. Singlefraction vs. Fractionated linac-based stereotactic radiosurgery for vestibular schwannoma: a single institution study. Int $J$ Radiation Oncology Biol Phys 2003; 56: 1390-6.

27. Kondziolka D, Nathoo N, Flickinger J et al. Longterm results after radiosurgery for benign intracranial tumors. Neurosurgery 2003; 53: 815-21.

28. Andrews D, Suárez 0, Goldman W et al. Stereotactic radiosurgery and fraccionated stereotactic radiotherapy for the treatment of acoustic schwannomas: comparative observations of 125 patients treated at one institution. Int $J$ Radiation Oncology Biol Phys 2001; 50: 1265-78.

29. Lundford L, Niranjan A, Flickinger J et al. Radiosurgery of vestibular schwannomas: summary of experience in 829 cases. $J$ Neurosurg (Suppl) 2005; 102: 195-9.

30. Wombra B, Muacevic A, Jess-Hempen A et al. Outpatient Gamma Knife surgery for vestibular schwannoma: definition of the therapeutic profile based on a 10-year experience. $J$ Neurosurg (Suppl) 2005; 102: 114-8.

31. Hasegawa T, Fustami SH, Katsumata SH et al. Stereotactic radiosurgery for vestibular schwannomas: analysis of 317 patients followed more than 5 years. Neurosurgery 2005; 57: 257-63.

32. Hasegawa T, KIDA Y, KobaYASHI T ET AL. Long-term outcomes in patients with vestibular schwannomas treated using Gamma Knife surgery: 10-years follow up. J Neurosurg (suppl). 2005; 102: 10-6.

33. Chung W, Liu K, Shiau Ch et al. Gamma Knife surgery for vestibular schwannoma: 10-years experience of 195 cases. J Neurosurg (Suppl) 2005; 102: 87-96.

34. Iwal Y, Yamanaka K, Shiotani M et al. Radiosurgery for acoustic neuromas: results of low-dose treatment. Neurosurgery 2003; 53: 282-7.

35. FLICKINGER J, KONDZIOLKA D AND LUNSFORD L. Dose and diameter relationships for facial, trigeminal and acoustic neurophaties following acoustic neuroma radiosurgery. Radiother Oncol 1996; 41: 215-9.

36. Flickinger J, Kondziolka D, Niranjan a et al. Acoustic neuroma radiosurgery with marginal tumor doses of 12 to $13 \mathrm{~Gy}$. Int $J$ Radiation Oncology Biol Phys 2004; 60: 225-30.

37. Van Eck A and Horstmann G. Increased preservation of functional hearing after Gamma Knife surgery for vestibular schwannoma. $J$ Neurosurg (Suppl) 2005; 102: 204-6.

38. Paek S, Chung H, Jeong $S$ et al. Hearing preservation after Gamma Knife stereotactic radiosurgery of vestibular schwannoma. Cancer 2005; 104: 580-90.

39. Massager N, Nissim 0, Delbrouck C et al. Role of intracanalicular volumetric and dosimetric parameters on hearing preservation after 
vestibular schwannoma radiosurgery. Int $J$ Radiation Oncology Biol Phys 2006; 64: 1331-40.

40. Massager N, Nissim 0, Delbrouck C et al. Irradiation of cochlear structures during vestibular schwannoma radiosurgery and associated hearing outcome. J Neurosurg 2007; 107: 733-9.

41. Ottaviani F, Bartolomeo C, Ventrella L et al. Hearing loss and changes in transient evoked otoacoustic emissions after Gamma Knife radiosurgery for acoustic neurinomas. Arch Otolaryngol Head Neck Surg 2002; 128: 130812.

42. Beegle R, Friedman W and Bova F. Effect of treatment plan quality on outcomes after radiosurgery for vestibular schwannoma. $J$ Neurosurg 2007; 107: 913-6.

43. Bush ML, Shinn JB, Young AB Jones RO. Long-term results in Gamma Knife radiosurgery for acoustic neuromas. Laryngoscope2008 (In press).

44. LeKSELL L. A note on the treatment of acoustic tumors. Acta Chir Scand 1971; 137: 763-5.

45. Koos, WT, Day JD, Matula C et al. Neurotopographic considerations in the microsurgical treatment of small acoustic neurinomas. J Neurosurg 1998; 88: 506-12.

46. Shaw E, Kline R, Gillin M et al. Radiation Therapy Oncology Group: Radiosurgery quality assurance guidelines. Int J Radiation Oncology Biol Phys 1993; 27: 1231-9.

47. Schell M, Bova F, Larson D, Leavitt D, Lutz W, Podgorsak E, Wu A. AAPM report № 54, Stereotactic Radiosurgery. Published for the American Association of Physicists in Medicine by the American Institut of Physics, 1995.

48. GaRDNER G, RoBERTSON J. Hearing preservation in unilateral acoustic neuroma surgery. Ann Otol Rhinol Laryngol 1988; 97: 55-66.
49. House J, Brackmann D. Facial nerve grading system. Aolaryngol Head Neck Surg 1985; 93: 146-7.

50. Szeifert GT, Flgarella-Branger D, Roche PH, RÉGIS J. Histopathological observations on vestibular schwannomas after Gamma Knife radiosurgery: the Marseille experience. Neurochirurgie 2004; 50: 327-37.

51. Szeifert GT, Kondziolka D, Atteberry DS, Salmon I, Rorive S, Levivier M, Lundford LD. Radiosurgical pathology of brain tumors: metastases, schwannomas, meningiomas, astrocytomas, hemangioblastomas. Prog Neurol Surg 2007; 20: 91-105.

52. O'Connor M, Mayberg M. Effects of radiation on cerebral vasculature: a review. Neurosurgery 2000; 46: 138-49.

53. ShIN M, UaKI K, KURITA $H$ et al. Malignant transformation of a vestibular schwannoma after Gamma Knife radiosurgery. Lancet 2002; 360: 309-10.

54. Hanabusa K, Morikama A, Murata T, Taki W. Acoustic neuroma with malignant transformation. Case report. J Neurosurg 2001; 95: 518-21.

55. De Ipolyı AM, Yang I, Buckley A et Al. Fluctuating response of a cystic vestibular schwannoma to radiosurgery: Case report. Neurosurg 2008; 62 (5): E1164-5.

56. Shuto T, Inomori S, Matsunaga $S$ et al. Microsurgery for vestibular schwanoma aflter Gamma Knife radiosurgery. Acta Neurochir (Wien). Feb 7, 2008 (Epub ahead of print).

57. Mathieu D, Kondziolka D, Flickinger JC, Niranjan A, Williamson R, Martin JJ, Lundsford LD. Stereotactic radiosurgery for vestibular schwanomas in patients with neurofibromatosis type 2: an analysis of tumor control, complications, and hearing preservation rates. Neurosurgery 2007; 60: 468-70.

Dirección: Dr. José Lorenzoni Santos

Departamento de Neurocirugía, Pontificia Universidad Católica de Chile Marcoleta 352, $2^{\circ}$ piso, Santiago, Chile. Teléfono: 3543465

E mail: jls@med.puc.cl - jglorenzoni@gmail.com 